\title{
Path Tracing Photons Oscillating Through Alternate Universes Inside a Black Hole
}

\author{
Deep Bhattacharjee \\ Research Head at AATWRI-EGSPL R\&D Directorate, Bhubeneshwar, Orissa, India \\ Email: itsdeep@live.com \\ Mobile: $+91-8282923025$ \\ $\left(21^{\text {st }}\right.$ March 2021)
}

\begin{abstract}
In case of the maximally rotating Black Holes $(\mathrm{BH})$ through Kerr-Neumann frames, or as described in Boyer-Lindquist coordinates metrics, the rotation axis of the BHs inputs a frame dragging effect i.e., relativistically a Lens-Thirring Precession that accelerates the photon trajectories oscillates with a shaped induced rotations through the ring singularity, between alternate universes, as a means of an induced geodesics that takes a sharp turning points back and forth provided, in the prograde photon sphere, due to magnetorotational instability, the path tracing of a photons circulates as a smooth fiber bundles over the event horizon curves, that when gets interpolate between mixed trajectories behaves as a geodesics and thus forms a smooth Jacobi-fields through Jacobi-lines by mutual intersection of geodesics over the photon sphere which when somehow gets leaked inside the event horizon, then gets sucked in with not sufficient escape velocity for retardation and trapped in the compact singularity, oscillating back and forth through alternate universes.
\end{abstract}

Keywords: Photon Spheres - Jacobi Lines - Turning Points - Photon Trajectories - Geodesics - Path Tracing Curves - Oscillation Points - Half-Librations

\section{Tangent Spaces \& Jacobi Fields}

Considering the event horizon as a boundary manifold $\mathcal{G}$ and the null rays over the prograde photon sphere being tangent to this manifold as $\mathcal{G}_{T}$, which assembles all the tangent vectors in $\mathcal{G}$, the disjoint union is given by,

$$
\begin{aligned}
\mathcal{G}_{T} & =\coprod_{x \in \mathcal{G}} T_{x} \mathcal{G} \\
& =\bigcup_{x \in \mathcal{G}}\{x\} \times T_{x} \mathcal{G} \\
& =\bigcup_{x \in \mathcal{G}}\{x, y\} \mid y \in T_{x} \mathcal{G} \\
& =\left\{(x, y) \mid x \in \mathcal{G}, y \in T_{x} \mathcal{G}\right\}
\end{aligned}
$$

Where $T_{x} \mathcal{G}$ denotes tangent space to $\mathcal{G}$ at the point $x$. Then, $T_{x} \mathcal{G}$ can be thought of as a pair $\langle x, y\rangle$ where $x$ is a point in $\mathcal{G}$ and $y$ is a tangent vector to $\mathcal{G}$ at $x$. Therefore, the natural projection from the tangent to the tangent space, here from the prograde photon sphere to the event horizon as,

$\pi: T_{x} \mathcal{G} \rightarrow \mathcal{G}$

Overlapping tangents, creates a smooth family of geodesics $\mathcal{D}_{\mathcal{T}}$ with $\mathcal{D}_{0}=\mathcal{D}$, then the Jacobi-fields could be represented as a function,

$T_{x} \mathcal{G}(t)=\left.\frac{\partial_{\mathcal{D}_{\mathcal{T}}}(t)}{\partial \mathcal{T}}\right|_{\mathcal{T}=0}$

The tangent vector field $T_{x} \mathcal{G}$ along a geodesic $\mathcal{D}_{\mathcal{T}}$ is said to be Jacobi field if it satisfies the Jacobi equation:

$\frac{\mathcal{D}_{\mathcal{T}}{ }^{2}}{d t^{2}} T_{x} \mathcal{G}(t)+R\left(T_{x} \mathcal{G}(t), T_{x} \mathcal{G}(t)\right) T_{x} \mathcal{G}(t)=0$

Where $\mathcal{D}$ is the covariant derivative, $R$ is the Riemann curvature tensor, $T_{x} \mathcal{G}(t)=d\left(T_{x} \mathcal{G}(t)\right) / d t$ is the tangent vector field, and $t$ is the parameter along the geodesic.

\section{Black Hole Inequalities \& Photon Trajectories}

Now considering a $\mathrm{BH}$ in the Boyer-Lindquist coordinates $(t, r, \theta, \varphi)$ with mass $M$ and spin coefficient of the angular momentum $J=M a$, the inner and outer horizons of the Kerr BH is given by $[1,2,3,4]$, $r_{ \pm}=M \pm \sqrt{M^{2}-a^{2}}, \quad$ where $0<a<M$

We find some important parameters as,

$\gamma=\frac{\ell}{E}, \quad \eta=\frac{K}{E^{2}}$

With the corresponding value names, as, $E$ is the energy at infinity, $\ell$ is the spin angular momentum about its rotational axis, $K$ is the Carter integral having an affine valued label as,

$K=p_{\theta}^{2}-\cos ^{2} \theta\left(a^{2} p_{t}^{2}-p_{\phi}^{2} \csc ^{2} \theta\right)$
$E=-p_{t}$
$\ell=p_{\phi}$

With $p^{\mu}$ denoting the 4-momentum of the photon provided $p^{t}>0$ having the time orientation where $p^{\mu}$ can be reconstructed as,

$\frac{\Sigma}{E} p^{r}= \pm_{r} \sqrt{\mathcal{R}(r)}$
$\frac{\Sigma}{E} p^{\theta}= \pm_{\theta} \sqrt{\Theta(\theta)}$
$\frac{\Sigma}{E} p^{\phi}=\left(\ell \operatorname{Csc}^{2} \theta-a E\right)+\frac{a}{\Delta}\left(E\left(r^{2}+a^{2}\right)-a \ell\right)$
$\frac{\Sigma}{E} p^{t}=a\left(\ell-a E \operatorname{Sin}^{2} \theta\right)+\frac{r^{2}+a^{2}}{\Delta}\left(E\left(r^{2}+a^{2}\right)-a \ell\right)$

Where, the induced "potentials" are,

$\mathcal{R}(r)=\left(r^{2}+a^{2}-a \lambda\right)^{2}-\Delta(r)\left[\eta+(\lambda-a)^{2}\right]$

$\Theta(\theta)=\eta+a^{2} \operatorname{Cos}^{2} \theta-\lambda^{2} \operatorname{Cot}^{2} \theta$

Then, the 4-velocity $x^{\mu}$, "Mino time" $\tau[5]$ and 4-momentum $p^{\mu}$ could be related as,

$\frac{d x^{\mu}}{d \tau}=\frac{\Sigma}{E} p^{\mu}$

To avoid singularities in the spherical coordinate systems, it has been assumed that, $0<\theta<\pi$ and by labeling $\sigma=\cos ^{2} \theta$, the value is given by [6],

$(1-\sigma) \Theta(\sigma)=\eta+\left(a^{2}-\eta-\lambda^{2}\right) \sigma-a^{2} \sigma^{2}$

Where $\eta+\left(a^{2}-\eta-\lambda^{2}\right) \sigma-a^{2} \sigma^{2}$ is a quadratic polynomial, having the roots $\sigma_{ \pm}$is given by, 
$\sigma_{ \pm}=\frac{1}{2}\left(1-\frac{\eta+\lambda^{2}}{a^{2}}\right) \pm \sqrt[2]{\triangle_{\theta}^{2}+\frac{\eta}{a^{2}}}$

The 4-roots of $\Theta(\theta)$ are given by $\arccos \left( \pm \sqrt{\sigma_{ \pm}}\right)$, or,

$$
\begin{aligned}
& \theta_{1}=\arccos \left(\sqrt{\sigma_{+}}\right) \\
& \theta_{2}=\arccos \left(\sqrt{\sigma_{-}}\right) \\
& \theta_{3}=\arccos \left(-\sqrt{\sigma_{-}}\right) \\
& \theta_{4}=\arccos \left(-\sqrt{\sigma_{+}}\right)
\end{aligned}
$$

Coinciding each roots, occurs at the singular intersecting points,

$$
\sigma_{+}=0, \quad \sigma_{-}=0, \quad \sigma_{+}=\sigma_{-}
$$

Motion is allowable in the region where,

$$
\sigma_{+}>0, \quad \sigma_{-}<1,
$$

Implies the bound forms as,

$$
\eta \geq=\left\{\begin{array}{rr}
0, & |\gamma| \geq a \\
-(|\gamma|-a)^{2}, & |\gamma| \leq a
\end{array}\right.
$$

Therefore, the elemental line $\eta=0$ of double roots admits two characteristics' of null-geodesics as,

A. $\quad(\eta>0)$ forming ordinary geodesics where the photon liberates between $\theta_{1}$ and $\theta_{4}$ taking the limit $\theta_{1}<\frac{\pi}{2}<\theta_{4}$ crossing the equatorial plane each time with a "positive potential" in them.

B. $(\eta<0)$ forming vertical geodesics where there are 4-real roots as, $\theta_{1}<\theta_{2}<\frac{\pi}{2}<\theta_{3}<\theta_{4}$ where the "positive potentials" roots in the Northern hemisphere as $\left(\theta_{1}, \theta_{2}\right)$ and "negative potentials" as $\left(\theta_{3}, \theta_{4}\right)$ in the Southern hemisphere, being liberated in between the turning points $\left(\theta_{1}, \theta_{2}\right)$ and $\left(\theta_{3}, \theta_{4}\right)$.

C. $(\eta=0)$ forming equatorial plane itself with no turning points in limited "A" type motion having roots $\theta_{1,4}$ and limited " $\mathrm{B}$ " type motion having roots $\theta_{2,3}$, with each value suffices to $\frac{\pi}{2}$.

For, Type "A" motion, there are 3 angular integrals reduced to a real elliptic form $\mathcal{O}_{\theta}, \mathcal{O}_{\phi}$ and $\mathcal{O}_{t}$, since $\sigma_{+} / \sigma_{-}<0$, the antiderivatives becomes $[1,6]$,

$$
\begin{aligned}
& \mathcal{O}_{\theta}=-\frac{1}{-\sigma_{-} a^{2}} \mathcal{F}\left(\arcsin \left(\frac{\cos \theta}{\sqrt{\sigma_{+}}}\right) \mid \frac{\sigma_{+}}{\sigma_{-}}\right) \\
& \mathcal{O}_{\phi}=-\frac{1}{-\sigma_{-} a^{2}} \xi\left(\sigma_{+} ; \arcsin \left(\frac{\cos \theta}{\sqrt{\sigma_{+}}}\right) \mid \frac{\sigma_{+}}{\sigma_{-}}\right) \\
& \mathcal{O}_{t}=-\frac{2 \sigma_{+}}{-\sigma_{-} a^{2}} E^{\prime}\left(\arcsin \left(\frac{\cos \theta}{\sqrt{\sigma_{+}}}\right) \mid \frac{\sigma_{+}}{\sigma_{-}}\right)
\end{aligned}
$$

Are real and smooth, where it has been defined,
$E^{\prime(\varphi \mid k)}:=\partial_{k} E(\varphi \mid k)=\frac{E(\varphi \mid k)-\mathcal{F}(\varphi \mid k)}{2 k}$

Therefore, the half-libration values with a tilde as,

$$
\begin{aligned}
& \widetilde{\sigma_{\theta}}=\int_{\theta_{-}}^{\theta_{+}} \frac{d \theta}{\sqrt{\Theta(\theta)}}=\frac{2}{\sqrt{-\sigma_{-} a^{2}}} \nabla\left(\frac{\sigma_{+}}{\sigma_{-}}\right) \\
& \widetilde{\mathcal{O}_{\phi}}=\int_{\theta_{-}}^{\theta_{+}} \frac{\csc c^{2} \theta}{\sqrt{\Theta(\theta)}} d \theta=\frac{2}{\sqrt{-\sigma_{-} a^{2}}} \xi\left(\sigma_{+} \mid \frac{\sigma_{+}}{\sigma_{-}}\right) \\
& \widetilde{\mathcal{O}_{t}}=\int_{\theta_{-}}^{\theta_{+}} \frac{\csc c^{2} \theta}{\sqrt{\Theta(\theta)}} d \theta=-\frac{4 \sigma_{+}}{\sqrt{-\sigma_{-} a^{2}}} E^{\prime}\left(\frac{\sigma_{+}}{\sigma_{-}}\right)
\end{aligned}
$$

Here, $\mathcal{O}_{\theta}=\tau$ is the Mino time, the polar motion $\theta_{\circ}(\tau)$ has Mino time period of $2 \widetilde{\mathcal{O}_{\theta}}$.

For, Type "B" motion, the geodesic would oscillate with a hemispheric motion $\mathcal{H}$ as,

$$
\mathcal{H}=\operatorname{sign}(\cos \theta)
$$

The motion lies, within the parameter $\theta_{-}<\theta_{+}$in the Northern hemisphere $\mathcal{H}=+1$, and $\theta_{-}>\theta_{+}$in the Southern hemisphere $\mathcal{H}=-1$ having the retardation points given by,

$\theta_{ \pm}=\arccos \left(\mathcal{H} \sqrt{\sigma_{ \pm}}\right)$

Such that, $\theta_{+,-}=\theta_{1,2}$ for $\mathcal{H}=+1$ and $\theta_{+,-}=\theta_{3,4}$ for $\mathcal{H}=-1$, thereby, again , there are 3 angular integrals reduced to a real elliptic form $\mathcal{O}_{\theta}, \mathcal{O}_{\phi}$ and $\mathcal{O}_{t}$, since $\sigma_{+} / \sigma_{-}>0$, the antiderivatives becomes $[1,6]$,

$\mathcal{O}_{\theta}=-\frac{\mathcal{H}}{\sqrt{\sigma_{-} a^{2}}} \mathcal{F}\left(\beta \mid 1-\frac{\sigma_{+}}{\sigma_{-}}\right)$

$\mathcal{O}_{\phi}=-\frac{\mathcal{H}}{\sqrt{1-\sigma_{-}} \sqrt{\sigma_{-} a^{2}}} \xi\left(\frac{\sigma_{+}-\sigma_{-}}{1-\sigma_{-}} ; \beta \mid 1-\frac{\sigma_{+}}{\sigma_{-}}\right)$

$\mathcal{O}_{t}=-\mathcal{H} \sqrt{\frac{\sigma_{-}}{a^{2}}} E\left(\beta \mid 1-\frac{\sigma_{+}}{\sigma_{-}}\right)$

Are real and smooth, with,

$\beta=\arcsin \sqrt{\frac{\cos ^{2} \theta-\sigma_{-}}{\sigma_{+}-\sigma_{-}}}$

The respective half-libration values are,

$$
\begin{aligned}
& \widetilde{\mathcal{O}_{\theta}}=\mathcal{H} \int_{\theta_{-}}^{\theta_{+}} \frac{d \theta}{\sqrt{\Theta(\theta)}}=\frac{1}{\sqrt{\sigma_{-} a^{2}}} \nabla\left(1-\frac{\sigma_{+}}{\sigma_{-}}\right) \\
& \widetilde{\mathcal{O}_{\phi}}=\mathcal{H} \int_{\theta_{-}}^{\theta_{+}} \frac{\csc c^{2} \theta}{\sqrt{\Theta(\theta)}} d \theta=\frac{1}{\sqrt{1-\sigma_{-}} \sqrt{\sigma_{-} a^{2}}} \xi\left(\frac{\sigma_{+}-\sigma_{-}}{1-\sigma_{-}} ; 1-\frac{\sigma_{+}}{\sigma_{-}}\right) \\
& \widetilde{\mathcal{O}_{t}}=\int_{\theta_{-}}^{\theta_{+}} \frac{\csc c^{2} \theta}{\sqrt{\Theta(\theta)}} d \theta=\sqrt{\frac{\sigma_{-}}{a^{2}}} E\left(1-\frac{\sigma_{+}}{\sigma_{-}}\right)
\end{aligned}
$$

Note: There is no radial potential as the motion is purely geodesic over the curve. Moreover, true radial potential is absurd as the intense gravity will curve the photon paths. 
Where $\widetilde{\mathcal{O}_{\theta}}$ denotes the Mino-time half period of the polar motion $\theta_{\circ}(\tau)$.

\section{Path Integral Formulations}

The photon trajectory should be real and smooth and if a particular trajectory $\psi(C)$ of a function $f_{0}$ where $C_{s}$ is the source and $C_{d}$ is the destination with the function parameter $\psi$ oscillates in the range $\psi \in\left[\psi_{+}, \psi_{-}\right]$then then the integral could be defined as,

$z=\int_{C_{s}}^{C_{d}} f_{0}(\psi(C))\left|\frac{d \psi}{d C}\right| d C$

Therefore, considering a segment over the respective turning points through the trajectory, where the integrand is simply $\pm \psi(C) d \psi$ with \pm the sign of $\left|\frac{d \psi}{d C}\right|$ where $\psi\left(C_{s}\right)=C_{s}$ and $\psi\left(C_{d}\right)=C_{d}$ (thereby switching points) the integral depends on the initial value $C_{s}$ and initial sign $\omega=\operatorname{sign}\left(\psi^{\prime}\left(C_{s}\right)\right)$, going to the final value $C_{d}$ with the turning points encountered along the way as $\mathcal{J}$, the relation suffice to,

$Z=Z\left(C_{s}, \omega, C_{d}, \mathcal{J}\right)$

The antiderivatives $h^{*}$ could be explained as,

$\frac{d h^{*}}{d C}=\psi(C)$

With the notional notation $h^{*}{ }_{i}=h^{*}\left(C=C_{i}\right)$ with $i \in\{s, d, \uparrow, \downarrow\}$ producing the integral as,

$\overline{\bar{z}}=\int_{C^{\downarrow}}^{C^{\uparrow}}(\psi(C)) d C=h^{* \uparrow}-h^{* \downarrow}$

Above denoting the half-libration path integral having a quasiperiodicity property as,

$z(\mathcal{J}+2)=Z(\mathcal{J})+2 \overline{\bar{Z}}$

This implicitly gives us 4-values as in order of,

$\omega=\uparrow 1, \quad \mathcal{J}=0: \quad Z=h^{*}\left(C_{d}\right)-h^{*}\left(C_{s}\right)$

$\omega=\downarrow 1, \quad \mathcal{J}=0: \quad Z=h^{*}\left(C_{d}\right)-h^{*}\left(C_{s}\right)$

$\omega=\uparrow 1, \quad \mathcal{J}=1: \quad z=-h^{*}\left(C_{s}\right)-h^{*}\left(C_{d}\right)-2 h^{*}(C)_{\uparrow}$

$\omega=\downarrow 1, \quad \mathcal{J}=1: \quad Z=h^{*}\left(C_{s}\right)+h^{*}\left(C_{d}\right)-2 h^{*}(C)_{\downarrow}$

Obtaining the relation as,

$z=\left\{\begin{array}{rr}\mathcal{J} \overline{\bar{z}}+\omega\left(h^{*}\left(C_{d}\right)-h^{*}\left(C_{s}\right)\right), & \text { J even } \\ \mathcal{J} \overline{\bar{Z}}+\omega\left(h^{* \uparrow}+h^{* \downarrow}-h^{*}\left(C_{d}\right)-h^{*}\left(C_{s}\right)\right), & \text { J odd }\end{array}\right.$

Which correctly reproduces the final path tracing photon in geodicity as,

$Z=\mathcal{J} \overline{\bar{Z}}+\omega\left[(-1)^{\jmath} h^{*}\left(C_{d}\right)-h^{*}\left(C_{s}\right)+\frac{1-(-1)^{\mathcal{J}}}{2}\left(h^{* \uparrow}+h^{* \downarrow}\right)\right]$

\section{Conclusion}

The photon trajectory has been considered devoiding of any coordinate singularity as mentioned before having the values, $(0<\theta<\pi)$, with a "potential" measuring through the Boyer-Lindquist coordinates $(t, r, \theta, \varphi)$, taking the 'angular' parameter as Boyer-Lindquist coordi- nates $(t, \theta, \varphi)$, neglecting the "radial" parameter Boyer-Lindquist coordinates $(r)$ because the 3 -potentials that suffice the angular momentum induced trajectory through the values of "ordinary geodesics" and vortical geodesics" where the later behave has an induced radial curves of null paths induced by relativistically frame dragging having the "affine-valued" functions $(\eta>0),(\eta=0),(\eta<0)$ through the "3-potentials of $\left(\mathcal{O}_{\theta}, \mathcal{O}_{\phi}, \mathcal{O}_{t}\right)$ along with half-librations $\left(\mathcal{O}_{\theta}, \overline{\mathcal{O}_{\phi}}, \mathcal{O}_{t}\right)$, focusing primarily the oscillations between alternate universes, having 'null coordinate singularity' but a 'way out' "ring" singularity through which the photons being trapped through the generators of the cone, sprouts out through the fountains making a way of rectifying the Lens-Thirring effect with an induced rotation that calibrates inside the event horizon having the escape velocity values $V_{\text {esc }} \sim 3,00,000 \mathrm{Km} / \mathrm{Sec}$ making the path tracing of the photon stays inside the $\mathrm{BH}$ as a permanent candidature. At the last, the "path integrals" have been computed for 4-affine valued parameters, along with turning points, $\left(C_{s}, \omega, C_{d}, \mathcal{J}\right)$ to provide a summation of all the path tracing as concerned in this paper.

\section{References:}

[1] Gralla, S. E., \& Lupsasca, A. (2020). Null Geodesics of the Kerr Exterior. arXiv:1910.12881v3 [gr-qc]

[2] Wei, S., Zou, Y., Liu, Y., \& Mann, R. B. (2019). Curvature radius and Kerr black hole shadow. arXiv:1904.07710v2

[3] Bhattacharjee, D. (2020). Solutions of Kerr Black Holes subject to Naked Singularity and Wormholes. Authorea, 14. https://doi.org/10.22541/au.160693414.46356832/v1

[4] Bhattacharjee, D. (2021). Deciphering Black Hole Spin, Inclination angle \& Charge From Kerr Shadow. ResearchGate.

[5] Mino, Y., (2003) "Perturbative approach to an orbital evolution around a supermassive black hole," Phys. Rev. D 67 no. 8,084027, arXiv:gr-qc/0302075 [gr-qc]

[6] Kapec, D., and Lupsasca, A., (2019) "Particle motion near high-spin black holes," arXiv e-prints; arXiv:1905.11406, arXiv:1905.11406 [hep-th].

Author declares that he does not have any competing interests as related to this paper.

ORCID ID - https://orcid.org/0000-0003-0466-750X 